\title{
Resigned Indifference: The importance of Cultural Competence Education
}

\author{
Markey, Kathleen; Tilki, Mary; Taylor, Georgina \\ Department of Nursing and Midwifery, University of Limerick, Ireland.
}

\begin{abstract}
This paper presents the findings of a PhD study, which explored how nurses' deal with their main concern when caring for patients from diverse cultural and ethnic backgrounds. Utilising theoretical sampling and the principles of a grounded theory approach, focus groups (n-10) and individual face-to-face interviews (n-30) were conducted with student and qualified nurses, in one region of Ireland. As data were collected, it was simultaneously analysed using constant comparative analysis during open, selective and theoretical coding. Uncertainty was the consistent main concern that emerged for participants in this study. Feelings of ambiguity of how to act were further influenced by a lack of knowledge, an awareness of potential ethnocentric beliefs and the culture of the organisation in which participants learn and work in. Resigned indifference explains how participants in this study dealt with their uncertainty when caring for patients from diverse cultures. It explains how participants adopted a range of disengagement strategies. Instead of doing what they sometimes knew to be right, participants adopted a range of disengagement strategies which were underpinned by a resigned indifference. The culture within the organisation allowed the disengagement strategies and indifference to also go un-noticed. As a result culturally insensitive care went unchallenged, often un-noticed and subsequently unchanged. The discomfort associated with providing culturally insensitive care was eased with shifting the blame to professional preparation or organisational constraints. As a consequence culturally insensitive care is sustained and perpetuated. This paper highlights the need for imaginative learning and teaching approaches that will replace uncertainty with curiosity and resilience, apathy with courage and commitment both at individual and organisational levels.
\end{abstract}

Keywords: Cultural Competence Education; Cultural diversity; Cultural Difference; Nursing; Nurse Education 


\section{Introduction}

There is a wealth of evidence in the literature which suggests that nurses experience a range of challenges when caring for patients from diverse cultural and ethnic backgrounds. However, there is a need to further understand how care is organised in healthcare settings as well as what influences nurses attitudes, practices and behaviour when providing quality care in a culturally appropriate way. There is also a need to explore how the culture of the organisation can help or hinder nurses' ability to provide culturally competent care. Although the current literature highlights the different complexities associated with culture competence, it fails to comprehensively identify the extent of the challenges experienced or explain in any depth how nurses address them in their daily practice. From reviewing the literature, it is evident that it remains unclear how nurses prioritise care needs of patients with different cultures and what influences these priorities. This paper presents some key findings from a PhD study (Markey 2017) which explored how nurses deal with their main concern when caring for patients from diverse cultural and ethnic backgrounds, in an Irish context. Drawing on findings, this paper makes a number of recommendations for cultural competence education in the classroom and practice setting.

\section{Background}

There is a wealth of international literature highlighting the importance of providing culturally competent care (Campinha-Bacote 2011; Jenks 2011) and more recently culturally competent compassion (Papadopoulos et al. 2016). However, global reports of culturally insensitive care continue. Cultural competence education is the most commonly reported initiative recommended within the literature as a means of addressing such issues. This call is not unique to nursing, as there are also increasing calls for effective cultural competence education in the preparation of other professionals (Whiteford and Wright StClair 2002; O' Shaughnessy and Tilki 2007). However, there remains a lack of consensus within the literature as to how cultural competence education should be structured and organised. This requires a deeper understanding as to what the daily challenges are for professionals during cross-cultural encounters and what influences their attitudes, behaviours and practices. Although there is a wealth of evidence within the literature describing various approaches to delivering effective cultural competence education, there is a lack of consensus with regards to the most effective approaches. It is striking how little is known about the challenges, problems and pitfalls of cultural competence education. Although this paper answers some of these questions, it raises other questions that require further consideration.

Feeling ill prepared to care for patients of different cultures has been widely reported as a challenge for nurses globally. Studies carried out in Ireland (Lyons et al. 2008; Tuohy et al. 
2008) and more globally (Jirwe et al. 2009; Starr and Wallace 2009) all highlight the difficulties nurses face when they feel ill prepared to care for different cultures. In particular these studies highlight how nurses feel unsure of how to act and as a consequence are generally fearful of getting it wrong. However, these studies fail to explore how nurses deal with these issues in their daily practice, suggesting the need for further research in the area. While there is an abundance of literature on cultural competence, there is a lack of empirical studies exploring how nurses learn and develop cultural competence on a daily basis. This study helps to fill this gap in our existing understanding. The contextual background to this study is reported on in Markey et al (2012).

\section{Methods}

This study adopted the principles of a classic grounded theory approach (Glaser 1992) aiming for emerging data grounded in participants' perspectives and experiences. Reasons for choosing a classic grounded theory approach over other grounded theory approaches are reported in Markey et al. (2014). Focusing on the main concern of participants and how they deal with it are key components of classic grounded theory (Glaser 1992). Ethical approval was granted from the University and Region Hospital ethics committees. Anticipating and addressing issues of ethical consideration was fundamental in every step of the research process.

In adopting a subtle realist ontological position, capturing accurately and honestly the views and experiences of participants, remaining non-judgemental, prolonged engagement with data and reflexivity were fundamental during data collection and analysis. Data were collected using focus groups (n-10) and individual face-to-face interviews (n-30) theoretically sampling student and qualified nurses, in one region of Ireland. It became clear that students' development of cultural competence was largely influenced by the qualified nurses on the ward, hence signposting the researcher to invite qualified nurses to participate in this study. As data were collected, it was simultaneously analysed using the classic grounded theory methodological principles of open, selective and theoretical coding. Data analysis began with line-by-line in vivo coding transcripts for patterns of behaviour, where key phrases as used by participants own words were noted (Glaser 1992). The data were coded in every way possible, by asking the following questions; what is actually happening in the data? What is the main concern faced by participants? How do participants deal with their concerns? As codes emerged they were clustered into categories. These categories formed the basis for further selective coding exploring how participants dealt with their main concern in practice. Mulling with data, memoing, mind mapping and renaming codes and categories many times were fundamental processes that assisted the conceptualisation of emerging data. Theoretical coding enabled the relationship 
of all the categories to be considered in a clear and distinct way and enabled the weaving back of the fractured data that occurred as a result of open and selective coding. The credibility of this study was evaluated using Glasers' (1998) criterion of fit, relevance, workability and modifiability. This theory fits the phenomenon, has workability in explaining how the main concern of participants is dealt with in daily practice, is modifiable with new data and has relevancy to the participants.

\section{Findings}

The findings describe the different challenges nurses face and how they dealt with them in their daily practice when caring for patients from diverse cultural and ethnic backgrounds. However, the main concern for participants was uncertainty. A number of factors influence the extent of the uncertainty experienced, such as lack of knowledge, ethnocentricity and the organisational culture. Participants consistently described feelings of uncertainty and as a result were fearful of "doing or saying the wrong thing" during cross-cultural encounters.

"I suppose that you would find it weird at the start and because of that, you are always worried about saying the wrong thing and putting your foot in it. Because you don't have the nitty gritty of the knowledge required" (Student Interview 11)

Participants were critical of the cultural competence preparation received during education and training. Although participants appeared to want further information and cultural knowledge, they made few attempts to find out for themselves. Instead, participants used a range of disengagement strategies as a means of dealing with the uncertainty experienced when caring for patients from diverse cultural and ethnic backgrounds.

\section{Disengagement Strategies}

Participants described how they used a raft of disengagement strategies as a means of dealing with their uncertainties and getting through the day. These disengagement strategies allowed them to disguise the extent of the uncertainties experienced, whilst enabling them to provide care to patients from diverse cultures. Participants also described hiding their uncertainties and knowledge limitations to patients and their peers as a means of selfprotecting. This was often described in the context of carrying out a performance and doing what was seen to be needed to get through the day with as little conflict as possible. 
"I do think we do stereotype in our heads...we may not........act it out. I was thinking it in my head. Now ...I didn't let it affect my care, I put my own views aside." (Student Interview 4)

Although nurses did not want to allow their uncertainties and ethnocentricities to affect the care they provided, they did not feel able to deal with these issues appropriately in their daily practice. In taking such an approach, ethnocentric values, stereotypes and prejudices remain unchallenged and unaltered. The following interview extract illuminates the consistent behaviour described by participants, whereby temporary measures were taken to disguise as opposed to challenge ethnocentricities and stereotypes. This demonstrates the dichotomy between what nurses' think at a personal level and what they feel they need to do to survive in clinical practice.

"You have values and beliefs, but you have to leave like your personality and personal views at the door or in your car because you have to act in a certain way. You can collect them again on your way out. It's the only way we can ensure the patient care is not affected" (Student Interview 6)

Participants described how they used a range of avoidance strategies to minimise contact with patients from diverse cultural and ethnic backgrounds as a means of dealing with their uncertainties and fears of causing offense unintentionally. When participants did have contact with patients, they described focussing on the physical aspects of their care only, which was often described as tasks at the determent of meaningfully engaging during crosscultural encounters.

"My worry of offending someone is taking over from my ability to care for them.. so often I avoid having contact with them as a precaution." (Student Interview 13)

The culture of the practice setting where nurses learn and work was described as routinizing care. Data highlights that routine assisted to ease the uncertainty experienced as it provided some guidance to their uncertainties. However, not disputing the value of such guidance, the routine described was often rigid, ethnocentric and task-orientated. Participants described various reasons why they needed to fit in with the organizational culture and keeping to the routine that was accepted, took precedence over providing person centered care. Although keeping to the routine helped ease the discomfort associated with feeling uncertain, it facilitated and allowed the disengagement strategies to go un-noticed.

"If I keep to the routine and do as everyone else is doing then it is less likely to illuminate or highlight the bits I don't know or have a clue about" (Student Interview 19) 
However, for others being part of the team required complying with practices and attitudes that they sometimes knew to be incorrect. The need to fit in superseded the need to fulfil moral, professional and legal obligations.

"we want to fit in...we want to kind of...don't want to be labelled as the kind of awkward nurse...or the one who is always challenging or questioning" (Qualified Nurse Interview 7)

As a result of adopting such disengagement strategies, there were various examples of culturally insensitive care provided albeit as a result of thoughtlessness, ignorance and unwitting prejudices, as exemplified by the following informant.

"But it's kind of subconscious....well that is what I think anyway nothing bad is intended” (Student Focus Group 2)

\section{Resigned Indifference}

Participants consistently articulated that culturally insensitive care was rarely intended; however there was a general failure to take action to improve the care described.

“We know it's wrong but we don't do anything about it.” (Qualified Focus Group 2)

There was also a lack of confidence to question their own or their colleagues' attitudes and practices. The fear of being rejected by their peers or failing clinical assessments took precedence over doing the right thing by their patients. Participants described "not wanting to rock the boat".

"Students will definitely not question or challenge poor practices as that would mean their competencies would be at risk if you know what I mean" (Student Interview 1)

However there was a sense of resigning to the fact that nothing could be done to improve the situation. This was contextualised into adopting various self-rationalising behaviours as a means of self-protecting and easing the discomfort associated with the implications of their actions or omissions. It was easier to blame the organisation for the failure to provide adequate resources, the lack of education and training received or the unprecedented nature of migration to Ireland. Not withstanding the real pressures nurses experience working in an increasingly complex and diverse healthcare setting, however sometimes such issues were sometimes used as an excuse for the substandard care described. Instead of exploring ways to improve practices and the care provided, participants became resigned to and accepting of the substandard care given to patients from diverse cultural and ethnic backgrounds. 
"It's not our faults, what they expect to happen if they don't provide us with the staff or interpreters to help us to communicate appropriately with the patient" (Qualified Nurse Interview 8)

Perhaps more concerning was the acceptance of it being acceptable at just doing an "ok job" and doing enough to get by.

"We treat all our patients the same regardless.... And it's...I think we are doing an OK job” (Qualified Nurse Interview 6)

\section{Conclusion and Discussion}

This paper presents an overview of the findings from a grounded theory study that explored how nurses deal with their main concern when caring for patients from diverse cultural and ethnic backgrounds. Although this study explored experiences and views of nurses in one region of Ireland, they may be applicable to other professions and contexts. Uncertainty was the consistent main concern that emerged for participants in this study. A number of factors influence the extent of the uncertainty such as lack of knowledge, ethnocentricity and the culture of the organization in which nurses learn and work. Feelings of uncertainty associated with caring for patients from different cultures have been reported by others (Jirwe et al 2009; Hart and Mareno 2014). However, what appears to be missing from this evidence is an explanation of how nurses deal with their uncertainties in practice. There also appears limited evidence explaining what influences nurse attitudes, practices and behaviors when caring for patients from different cultures. This study therefore extends our understanding as it explains how nurses deal with the uncertainty experienced in their daily practice and helps us understand the factors that influence nurses' attitudes, behaviours and practices during cross-cultural encounters.

Resigned indifference helps explain how and why participants in this study used a range of disengagement strategies when caring for patients from diverse cultural and ethnic backgrounds. It explains how participants reduce the personal discomfort associated with not doing what they sometimes knew to be right, by self-rationalising, justifying their behaviour and shifting the blame for the substandard care described. It explains how nurses self-preserve in times of ambiguity and feel unable to deal appropriately with the daily dilemmas faced in healthcare settings today. Despite participants articulating awareness of the moral, professional and legal responsibilities on nurses to provide quality care, culturally insensitive attitudes and practices were frequently described. However these went unchallenged and subsequently unchanged, raising further questions as to why such approaches to care go unnoticed and remain unchallenged both in the classroom and practice setting. The findings also raise questions as to why nurses feel it acceptable to 
provide sub standard care to patients from different cultures and why they choose to do sometimes what they know to be incorrect. Although such reports are not unique to caring for diverse cultures (Francis Report 2012), the findings highlight the need for nurses to stop seeing cultural aspects of care as separate. While acknowledging the individual contexts and the constraints experienced, the ease at doing enough to get by, not rocking the boat and shifting the blame are significant findings that warrant consideration. Although the findings do not imply that participants were weak willed and unable to think for themselves, the culture of the organisation is one that does not encourage or support assertiveness or the challenging of attitudes and practices. It was easier to comply with accepted practices than to risk being rejected or suffer the consequences of challenging those in authority. Therefore, this study emphasises the need to pay careful attention to how cultural competence is taught, learnt and most importantly applied in practice, rather than narrowly considering curriculum content in isolation.

The findings highlight the need to re-shape the core values underpinning curriculum design and development, to ensure a continuous commitment to, and reflection upon personal and professional attitudes, behaviours and practices. Taking responsibility for learning and development must replace the ease at shifting the blame and self-rationalising behaviour. Opportunities to explore values underpinning culturally sensitive care demand rethinking educational philosophies and learning and teaching methodologies both in the classroom and during clinical placements. This does not necessarily require major structural changes but instead requires a shift in thinking. Developing independent and enquiry based learning skills is essential in replacing the uncertainty with curiosity. The findings emphasise the need to help nurses rehearse the skills of questioning, clarifying and challenging when something is not right. They need to understand ethical, legal and professional imperatives and develop courage to challenge ethnocentricity and individual and collective indifference. The challenge for nurse educators is to find the balance between developing knowledgeable doers and emotionally intelligent practitioners that have a greater self-awareness of individual and organizational attitudes that may hinder culturally sensitive care.

\section{References}

Campinha-Bacote, J. (2011) 'Delivering Patient-Centred Care in the Midst of a Cultural Conflict: The Role of Cultural Competence' OJIN: The Online Journal of Issues in Nursing, 16(2), Manuscript 5. DOI: 10.3912/OJIN.Vol16No02Man05 (accessed Dec 5 2016).

Francis, R. (2013) Report of the Mid Staffordshire NHS Foundation Trust Public Inquiry. www.midstaffspublicinquiry.com/report (accessed: November 21 2016).

Glaser, B. (1992). Basics of grounded theory analysis, California, CA Sociology Press. 
Glaser, B. (1998) Doing Grounded Theory; Issues and Discussions, California, CA Sociology Press

Jenks, A.C. (2011) "From "List of Traits" to "Open-Mindedness": Emerging Issues in Cultural Competence Education', Cultural Medical Psychiatry, 35(2), 209-235.

Jirwe, M., Gerrish, K., Keeney, S. and Emami, A. (2009). 'Identifying the Core Components of Cultural Competence: Findings from a Delphi Study', Journal of Clinical Nursing, 18, $2622-2634$.

Hart, P.L and Mareno, N. (2014) 'Cultural Challenges and Barriers through the Voices of Nurses', Journal of Clinical Nursing, 23 (15-15), 2223-2232.

Lyons, S. M., O'Keefe, F., Clarke, A. T. and Staines, A. (2008). 'Cultural Diversity in the Dublin Maternity Services: The Experiences of Maternity Services Providers When Caring for Ethnic Minority Women', Ethnicity and Health, 13 (3), 261-276.

Markey, K., Tilki, M. and Taylor, G. (2012) 'Strategies to Surmount the Potential Barriers to Providing Discriminatory Care in Irish Healthcare Settings', Contemporary Nurse, 40 (2), 256-263.

Markey, K., Tilki, M. and Taylor, G. (2014) 'Reflecting on the Challenges of Choosing and Using a Grounded Theory Approach', Nurse Researcher, 22(2), 16-22.

Markey, K. (2017) 'A Grounded Theory Study of Nurses' Experiences of Caring for patients from Diverse Cultures", Unpublished thesis, Middlesex University.

O' Shaughnessy, D. and Tilki, M. (2007). 'Cultural Competency in Physiotherapy: A Model for Training', Physiotherapy, 93, 69-77.

Papadopoulos, I., Shea S., Taylor, G., Pezzella, A. and Foley, L. (2016) 'Developing Tools to Promote Culturally Competent Compassion, Courage, and Intercultural Communication in Healthcare', Journal of Compassionate Health Care, 3(2), 1-20, DOI: 10.1186/s40639-016-0019-6.

Starr, S. and Wallace, D.C. (2009). 'Self-reported Cultural Competence of Public Health Nurses in a South-eastern U.S. Public Health Department', Public Health Nursing, 26(1), 48-57.

Tuohy, D., McCarthy, J., Cassidy, I. and Graham, M. M. (2008). 'Educational Needs of Nurses When Nursing People of a Different Culture in Ireland', International Nurse Review, 55 (2), 164-170.

Whiteford, G. and Wright St-Clair (2002). 'Being Prepared for Diversity in Practice: Occupational Therapy Students' Perceptions of Valuable Intercultural Learning Experiences', The British Journal of Occupational Therapy, 65(3), 129-137 